\title{
The Demand for Inpatient and ICU Beds for COVID-19 in the US: Lessons From Chinese Cities
}

\section{Citation}

Ruoran, Li, Caitlin Rivers, Qi Tan, Megan B Murray, Eric Toner, and Marc Lipsitch. The Demand for Inpatient and ICU Beds for COVID-19 in the US: Lessons From Chinese Cities (March 2020).

\section{Permanent link}

http://nrs.harvard.edu/urn-3:HUL.InstRepos:42599304

\section{Terms of Use}

This article was downloaded from Harvard University's DASH repository, and is made available under the terms and conditions applicable to Other Posted Material, as set forth at http:// nrs.harvard.edu/urn-3:HUL.InstRepos:dash.current.terms-of-use\#LAA

\section{Share Your Story}

The Harvard community has made this article openly available.

Please share how this access benefits you. Submit a story. 
2 cities

4 Ruoran Li M.Phil., ${ }^{1}$ Caitlin Rivers Ph.D., ${ }^{2}$ Qi Tan M.D. Ph.D., ${ }^{3,4}$ Megan B Murray D.PH. M.D., ${ }^{3}$

5 Eric Toner M.D., ${ }^{2}$ Marc Lipsitch D.Phil. ${ }^{1}$

6

7 1. Center for Communicable Disease Dynamics, Department of Epidemiology, Harvard T.H.

8 Chan School of Public Health, Boston, Massachusetts, USA

9 2. Johns Hopkins Center for Health Security and the Department of Environmental Health and

10 Engineering, Johns Hopkins Bloomberg School of Public Health, Baltimore, Maryland, USA

11 3. Department of Global Health and Social Medicine, Harvard Medical School, Boston,

12 Massachusetts, USA

13 4. Department of Respiratory and Critical Care Medicine, The First Affiliated Hospital of Nanjing

14 Medical University, Nanjing Medical University, Nanjing, China

16 Corresponding author: Ruoran Li, rul612@mail.harvard.edu, Department of Epidemiology,

17 Harvard T.H. Chan School of Public Health, 677 Huntington Ave, Boston, MA 02115

19 Keywords: outbreak, emerging infectious disease, coronavirus, disease control,

20 nonpharmaceutical interventions, critical care, hospitalization, vulnerable populations

22 Word count: 2210

23

24

25 


\section{Key points}

27 Question: What hospital capacity is needed to respond to outbreaks of SARS-CoV-2 in US

28 cities, and how does this depend on intervention timing?

29 Findings: If a Wuhan-like outbreak were to occur, 24.5 inpatient beds and 2.6 ICU beds per

3010,000 adults would be needed during the peak epidemic; earlier intervention vastly reduces

31 this.

32 Meaning: Strict disease control strategies are needed early to mitigate the demand for inpatient 33 and ICU beds.

34

35 


\section{Abstract}

37 Importance: Sustained spread of SARS-CoV-2 has happened in major US cities. Capacity

38 needs in Chinese cities could inform the planning of local healthcare resources.

39 Objective: To describe and compare the intensive care unit (ICU) and inpatient bed needs for

40 COVID-19 patients in two Chinese cities. To estimate the peak ICU bed needs in US cities if a

41 Wuhan-like outbreak occurs.

42 Design: Observational study

43 Setting: Wuhan and Guangzhou, China

44 Participants: Confirmed COVID-19 patients in Wuhan and Guangzhou from January 10 to

45 February 29, 2020.

46 Exposure(s): Timing of disease control measures in relation to the timing of SARS-CoV-2

47 community spread.

48 Main Outcome(s) and Measure(s): Total number of critical and severe patient-days and the

49 peak number of critically- and severely-ill patients over the study period.

50 Results: In Wuhan, strict disease control measures were implemented six weeks after

51 sustained local transmission of SARS-CoV-2. Between January 10 and February 29, COVID-19

52 patients accounted for an average of 637 ICU patients and 3,454 serious inpatients on each

53 day. During the epidemic peak, 19,425 patients (24.5 per 10,000 adults) were hospitalized,

549,689 (12.2 per 10,000 adults) were considered to be in serious condition, and 2,087 patients

55 (2.6 per 10,000 adults) needed critical care per day. In Guangzhou, strict disease control

56 measures were implemented within one week of case importation. Between January 24 and

57 February 29, COVID-19 accounted for an average of 9 ICU patients and 20 inpatients on each

58 day. During the epidemic peak, 15 patients were in critical condition, and 38 were classified as

59 serious. If a Wuhan-like outbreak were to happen in a US city, the need for healthcare

60 resources may be higher in cities with a higher prevalence of vulnerable populations. 
61 Conclusions and Relevance: Even after the lockdown of Wuhan on January 23, the number of

62 seriously ill COVID-19 patients continued to rise, exceeding local hospitalization and ICU

63 capacities for at least a month. Plans are urgently needed to mitigate the effect of COVID-19

64 outbreaks on the local healthcare system in US cities.

65

66

67

68

69

70 


\section{Introduction}

In the two months since the first report of four cases of atypical pneumonia in Wuhan, Hubei, China on December 27, 2019, ${ }^{1}$ the cumulative number of confirmed cases of COVID-19 in the city has risen to 49,122 , with 2,195 deaths from the disease by the end of February, $2020 .^{2}$ On January 23 , Wuhan city shut down in response to the fast-evolving epidemic. All public transportation within, to and from the city was suspended, and residents were barred from leaving. An estimated nine million people remained in the city after the lockdown. ${ }^{3}$ Strict social distancing measures were also implemented, including the compulsory wearing of facemasks in public.

During the early phase of the response in Wuhan, the number of patients overwhelmed local fever clinics and hospitals designated to receive COVID-19 patients. The media reported a significant shortage of hospital beds, intensive care unit (ICU) beds, and other healthcare resources. By February 12, over eighteen thousand health care workers were sent to Wuhan from other parts of China to help with the coronavirus response. ${ }^{4}$ Forty eight hospitals (including two new hospitals built specifically for COVID-19 patients) and over 26,000 inpatient beds were designated for the isolation and treatment of patients with confirmed SARS-CoV2. Quarantine centers with over 13,000 total beds were also established to isolate confirmed patients with milder illnesses. By the end of February, the local government reported that "finally patients don't need to wait for beds. Now the beds are waiting for patients." ${ }^{5}$

With human-to-human transmission now established in other countries, mitigating the potential impact of COVID-19 on local healthcare systems is a top priority. A recent clinical study from China reported that $81 \%$ of patients in whom SARS-CoV-2 is detected experience mild disease, $14 \%$ severe disease and $5 \%$ critical disease. ${ }^{1}$ However, questions still remain as to the proportion of asymptomatic patients and the clinical course of the disease, preventing accurate prediction of hospitalization and ICU needs using transmission models. 
Here, we describe the ICU and hospitalization needs for COVID-19 in two Chinese

97 cities: Wuhan, the epicenter of China's outbreak, and Guangzhou, a Chinese metropolis that

98 experienced early importation of cases. As with all Chinese cities, strict social distancing

99 measures and contact tracing and quarantine protocols were implemented since late January in

100 Guangzhou, which resulted in much smaller outbreak size than Wuhan. Describing and

101 comparing the resource needs in both cities may serve as benchmarks to help other large

102 metropolises in the world prepare for potential outbreaks.

103

104 Methods

105 We extracted and estimated confirmed COVID-19 case counts for severe and critical

106 cases from Wuhan and Guangzhou from situation updates from Chinese national and local

107 health commissions. We extracted the number of designated COVID-19 beds and

108 hospitalizations from the Wuhan Municipal Health Commission website.

A confirmed COVID-19 case was considered severe if the patient experienced at least

110 one of the following: dyspnea, respiratory frequency $\geq 30 /$ minute, blood oxygen saturation $\leq 93 \%$,

111 arterial blood oxygen partial pressure $(\mathrm{PaO} 2)$ to oxygen concentration $(\mathrm{FiO} 2)$ ratio $<300 \mathrm{mmHg}$,

112 and/or a pneumonia patient showing significant progression of lesions infiltrating $>50 \%$ of the

113 lung field on chest imaging within 24-48 hours. A confirmed patient was considered to be critical

114 if he/she experienced respiratory failure demanding invasive and/or non-invasive ventilation for

115 respiratory support, septic shock, and/or with multiple organ dysfunction/failure demanding

116 intensive care. ${ }^{6,7}$ These definitions have been more detailed with revisions of the Chinese

117 diagnostic and treatment guidelines. In this study, we used the term serious patients to describe

118 severe and critical patients collectively. We estimated the number of prevalent severe and

119 critical cases cross-sectionally per day - allowing for the fact that patients could move in and out

120 of these categories over the course of their disease. 
We extracted Wuhan city and Hubei province COVID-19 data between January 10 and

122 February 29, including the numbers of confirmed cases, new cures, new deaths, severe cases, 123 critical cases, serious cases (a sum of severe and critical cases), cumulative cures, cumulative 124 deaths, cumulative confirmed cases, and currently confirmed cases (cumulative confirmed 125 cases - deaths - cures). If official sources did not have data for variables on some dates, we 126 calculated the number of cases based on the relationships between variables. Because Wuhan 127 did not systematically report the number of severe and critical cases, we estimated these 128 numbers by assuming that the proportions of serious and critical cases out of all currently 129 confirmed cases was the same in Wuhan as in the rest of Hubei. For the dates when it was not 130 possible to estimate the severe and critical case counts using the above methods (January 18, 13125 , and 27), we assumed the number of severe and critical cases on those dates were the 132 same as reported for the previous day.

133 For Guangzhou, we extracted the city's case count on the number of confirmed, severe, 134 clinical, and cured cases and deaths for each day between January 24 and February 29.

Statistical analysis

We summed the total patient-days under critical and/or severe condition to estimate the 139 total ICU-days and serious-inpatient-days. We plotted the raw number of patients in critical and 140 severe conditions and patients hospitalized on each day for Wuhan and Guangzhou, and 141 estimated the proportion of hospitalization and ICU admission per 10,000 adults based on the 142 assumption that there were 9 million people present in Wuhan during the lockdown, ${ }^{3}$ of whom $14388.16 \%$ were age 15 or above (2010 census for cities in Hubei province), and 14.9 million 144 present in Guangzhou of whom $82.82 \%$ were age 15 or above (Guangdong statistical bureau). 145 
147 disease at the peak of a Wuhan-like outbreak in the 30 most populous US cities by assuming 148 that the effect of age and comorbidity on patient outcomes would be the same as their effect on

149 COVID-19 mortality as derived from case reports from China until February $11 .{ }^{8}$ Specifically, we 150 estimated the stratum-specific critical care rate in Wuhan by assuming that the risk factor for 151 being in critical care is the same as that for death (age and comorbidities, e.g. hypertension). ${ }^{8}$

152 We estimated the probability of being in critical condition at the peak of the epidemic in each 153 age and hypertension stratum using the COVID-19 mortality rate ratios for age and 154 hypertension ${ }^{8}$ and the proportion of Wuhan population in each stratum. The hypertension 155 prevalence in adults in Wuhan was estimated as $25.7 \%,{ }^{9}$ and the proportion of the population 156 aged over 65 years $14.1 \% .{ }^{10}$ We then applied these stratum-specific critical care rate to the 157 population structures in US cities based on the crude hypertension prevalence in adults in $1582017^{11}$ and the proportion of adult population over 65 years of age in these cities. ${ }^{12}$

\section{Results}

In Wuhan, COVID-19 accounted for a total of 32,486 ICU-days and 176,136 seriousinpatient-days between January 10 and February 29 (Figure 1), an average of 637 ICU patients and 3,454 serious inpatients on each day over that 51 day period. During the peak of the epidemic from mid to late February, a maximum of 19,425 patients (24.5 per 10,000 adults) were hospitalized, 9,689 patients (12.2 per 10,000 adults) were considered to be in "serious" condition, and 2,087 patients (2.6 per 10,000 adults) needed critical care per day. In Guangzhou, COVID-19 accounted for a total of 318 ICU-days and 724 inpatient-days

169 between January 24 and February 29 (Figure 2), an average of 9 ICU patients and 20

170 inpatients during that 37 day period. During the peak of the epidemic (early February), 15

171 patients were in critical condition, while 38 were hospitalized and classified as serious. Unlike 
172 Wuhan, where patients with mild COVID-19 disease were isolated in quarantine centers and not

173 in designated hospitals, all confirmed patients in Guangzhou were hospitalized until cure. The

174 maximum number of hospitalizations in Guangzhou on any day was 271 patients.

175 The projected number of prevalent critically ill patients at the peak of a Wuhan-like

176 outbreak in US cities ranges from 2.1 to 4.0 per 10,000 adults when we took into account of the

177 difference in age distribution (Figure 3, top), and from 2.6 to 4.9 per 10,000 adults when we

178 took into account of the differences in comorbidity (hypertension) prevalence (Figure 3,

179 bottom).

180

181 Discussion

182

Even after the lockdown of Wuhan on January 23, the number of seriously ill COVID-19

184 patients continued to rise, exceeding local hospitalization and ICU capacities for at least a

185 month. During the peak of the Wuhan epidemic in February, nearly 20,000 COVID-19 patients

186 were hospitalized simultaneously, with 10,000 in severe or critical conditions. If a Wuhan-like

187 outbreak were to take place in a US city, even with strong social distancing and contact tracing

188 protocols as strict as the Wuhan lockdown, hospitalization and ICU needs from COVID-19

189 patients alone may exceed current capacity. The need for healthcare resources may be higher

190 in some US cities where there is a higher prevalence of vulnerable populations (age and

191 comorbidity) than in Wuhan.

192 Exceeding healthcare capacity may increase the community spread of SARS-CoV-2. In

193 Wuhan, home isolation and quarantine were used in the early phase of the epidemic to alleviate

194 the demand in healthcare resources. However, because of the exponential increase of the

195 number of patients who developed serious illness but could not be hospitalized due to capped

196 capacity, secondary transmission in the community continued as patients and their household

197 contacts moved between hospitals seeking care. 

being able to get access to a ventilator, which would lead to an increased case fatality ratio. By

200 the end of February, Wuhan's case fatality ratio was 4.5\%,3.2\% for the rest of Hubei province, 201 and for the rest of China, where healthcare capacity was not exceeded due to strong social 202 distancing and contact quarantine measures in the early phase of the epidemic (such as 203 Guangzhou), $0.8 \% .{ }^{13}$ One contributing factor to the lower case fatality ratio in the rest of China 204 may be higher case ascertainment than Wuhan during the early phase of the epidemic. In both Wuhan and Guangzhou, the lockdowns did not lead to immediate downturns in 206 the demand for hospitalization or the number of serious cases; rather, the peak in these 207 measures occurred approximately a month after the lockdown in Wuhan, and two weeks after 208 the lockdown in Guangzhou. This delay reflects the potentially long time from infection to severe 209 and critical conditions as many COVID-19 patients who eventually require ICU care present 210 initially as having only mild symptoms, ${ }^{14}$ and even longer time to discharge or death, ${ }^{15}$ resulting 211 in the accumulation of hospitalized cases long after the downturns in the community spread. In 212 Wuhan, the longer delay may also reflect the ongoing transmission after the lockdown described 213 above, which itself resulted from the overloading of the healthcare system.

214 This study has several limitations. We relied on officially reported statistics, which may 215 not represent the change of actual case counts over time, but rather a reflection of testing and 216 hospitalization capacity. The trend in Wuhan of the number of serious cases and 217 hospitalizations is thus not reflective of actual need, but rather the trend in maximum capacity of 218 the Wuhan system in diagnosis and treatment. We are therefore more confident of the 219 hospitalization and serious case counts in Wuhan after mid February, and in Guangzhou, where 220 excess capacities in diagnosis and treatment were reported based on both official and unofficial 221 sources. In addition, our projection of the ICU bed needs in US cities does not take into account 222 scenarios where local transmission may differ from that of Wuhan. 
The contact rate in Wuhan during the early phase of the epidemic may have been much

224 higher than what we expect to occur in US cities because of the increased number of social 225 contacts that occured in Wuhan due to the Lunar New Year celebrations. If social distancing 226 measures are effectively implemented early in US cities, the growth of the epidemic may be 227 delayed. But it is also possible that US cities may not be able to implement the extreme social 228 distancing measures that were put into place later in the epidemic in Wuhan. Therefore, the 229 actual number of hospital and ICU beds that will be needed over the course of a COVID-19 230 outbreak in a US city is impossible to estimate precisely. Our estimated capacity needs based 231 on a "Wuhan-like" outbreak could be a benchmark for what healthcare providers would expect 232 to see during the first three months of a local COVID-19 epidemic.

233 Historical evidence has shown that in 1918, US cities which imposed nonpharmaceutical 234 interventions early in the epidemic course and maintained their interventions over a long period 235 had lower peaks and fewer total cases of pandemic influenza than those which waited. ${ }^{16,17}$ Our 236 comparison of Wuhan and Guangzhou, although it is only two cities, dramatically illustrates the 237 same relationship of early intervention leading to lower epidemic sizes and peaks. The future 238 course of these epidemics and others around the world, of course, depends on the ability to 239 maintain burdensome control measures over an extended period.

240 In several countries with high-performing healthcare systems where SARS-CoV-2

241 transmission has been established earlier, both supplies of personal protective equipment in 242 hospitals and the availability of services has been problematic for COVID-19 care, and in all 243 locations, ICU bed capacity is limited. ${ }^{18}$ Plans are urgently needed to mitigate the effect of 244 COVID-19 outbreaks on the local healthcare system. 
1. Wu Z, McGoogan JM. Characteristics of and Important Lessons From the Coronavirus Disease 2019 (COVID-19) Outbreak in China: Summary of a Report of 72314 Cases From the Chinese Center for Disease Control and Prevention. JAMA. February 2020. doi:10.1001/jama.2020.2648 Commission. http://wjw.wuhan.gov.cn/front/web/showDetail/2020030109892. Accessed $254 \quad$ March 7, 2020.

3. chinanews.com. Wuhan Mayor: new coronavirus pneumonia cases may increase another 1000. chinanews.com. https://m.chinanews.com/wap/detail/zwsp/gn/2020/0127/9070530.shtml. Published January 27, 2020. Hubei. Hubei Daily. http://hbrbshare.hubeidaily.net/hbshare/news/detail_index.html?contentType=5\&contentld= 649546\&cld=0. Published February 13, 2020.

5. Changjiang Daily. Three steps for Wuhan to achieve "beds waiting for patients." wuhan.gov.cn. http://www.wh.gov.cn/2019_web/whyw/202003/t20200303_305956.html.

$264 \quad$ Published March 3, 2020. Accessed March 7, 2020.

6. WHO-China Joint Mission. Report of the WHO-China Joint Mission on Coronavirus Disease 2019 (COVID-19).; 16-24 February 2020. https://www.who.int/docs/defaultsource/coronaviruse/who-china-joint-mission-on-covid-19-final-report.pdf. (7th version, trial) 新型冠状病毒肺炎诊疗方案（试行第七版）. 
$2719,2020$.

272 8. Novel Coronavirus Pneumonia Emergency Response Epidemiology Team. The 273 epidemiological characteristics of an outbreak of 2019 novel coronavirus diseases (COVID274 19) in China. China CDC Weekly. 2020;2:1-10.

9. 代娟, 龚洁, 杨念念, 赵原原, 严亚琼. 武汉市城区居民高血压与糖尿病流行特征及慢性病危 276 险因素知晓情况. Chinese Journal of Prevention and Control of Chronic Diseases. $277 \quad 2019 ; 27(9): 663-671$.

278 Wuhan Municipal Health Commission. 2018 年武汉市人口老龄化形势分析.; 279 2019. http://wjw.wuhan.gov.cn/upload/file/20190301/1551431274814033488.pdf.

11. Centers for Disease Control and Prevention. 500 Cities Project: Local data for better health. https://www.cdc.gov/500cities/index.htm. Published December 5, 2019. Accessed March 8, 2020.

12. U.S. Census Bureau QuickFacts: United States. Census Bureau QuickFacts. https://www.census.gov/quickfacts/fact/table/US/PST045219. Accessed March 8, 2020. the People's Republic of China.

287 http://www.nhc.gov.cn/xcs/yqtb/202003/9d462194284840ad96ce75eb8e4c8039.shtml. $288 \quad$ Accessed March 7, 2020. 
15. Sanche S, Lin YT, Xu C, Romero-Severson E, Hengartner N, Ke R. The Novel Coronavirus, 2019-nCoV, is Highly Contagious and More Infectious Than Initially Estimated. Epidemiology. February 2020. doi:10.1101/2020.02.07.20021154

16. Hatchett RJ, Mecher CE, Lipsitch M. Public health interventions and epidemic intensity during the 1918 influenza pandemic. Proc Natl Acad Sci U S A. 2007;104(18):7582-7587.

17. Markel H, Lipman HB, Navarro JA, et al. Nonpharmaceutical interventions implemented by US cities during the 1918-1919 influenza pandemic. JAMA. 2007;298(6):644-654.

18. Legido-Quigley H, Asgari N, Teo YY, et al. Are high-performing health systems resilient against the COVID-19 epidemic? Lancet. 2020;0(0). doi:10.1016/S01406736(20)30551-1

19. Carr BG, Addyson DK, Kahn JM. Variation in critical care beds per capita in the United States: implications for pandemic and disaster planning. JAMA. 2010;303(14):13711372.

20. Wunsch H, Wagner J, Herlim M, Chong DH, Kramer AA, Halpern SD. ICU Occupancy and Mechanical Ventilator Use in the United States. Critical Care Medicine. 2013;41(12):2712-2719. doi:10.1097/ccm.0b013e318298a139

21. American Hospital Association. Trendwatch Chartbook 2018: Trends Affecting Hospitals and Health Systems.; 2018. https://www.aha.org/system/files/2018-07/2018-ahahttps://www.census.gov/data/tables/2018/demo/age-and-sex/2018-age-sex- 
315 composition.html. Accessed March 8, 2020.

$31623 . \quad$ Fast Facts on U.S. Hospitals, 2020. American Hospital Association.

317 https://www.aha.org/statistics/fast-facts-us-hospitals. Accessed March 8, 2020.

318 


\section{Funding and acknowledgments}

321

322 ML was supported by Award Number U54GM088558 from the National Institute Of General

323 Medical Sciences and Centers for Disease Control and Prevention (CK000538-01) coop

324 agreement. The content is solely the responsibility of the authors and does not necessarily

325 represent the official views of the National Institute Of General Medical Science, the National

326 Institutes of Health or the CDC.

327

328 We thank the entire Center for Communicable Disease Dynamics team for providing a

329 supportive environment for this work. We thank Dr. Helen Jenkins for critical feedback on data

330 visualization. $R L$ thanks her fellow doctoral students for their support.

331

332 
Figure legends:

Figure 1. Burden of serious COVID-19 disease in Wuhan and Guangzhou, China structure. $^{22}$

Figure 2. Estimate number of critically ill patients at the peak of a Wuhan-like outbreak in cities in the US, per 10,000 adults Bottom: taking into account of the proportion of population with hypertension Wuhan: 2.6 per 10,000 adults were critically ill at the peak of the COVID-19 epidemic, with a crude hypertension prevalence of $25.7 \%$ among adults (rate ratio for critical illness=6.9), and $15.9 \%$ adults over 64 years of age (rate ratio for critical illness=7.2). 


\section{Wuhan}

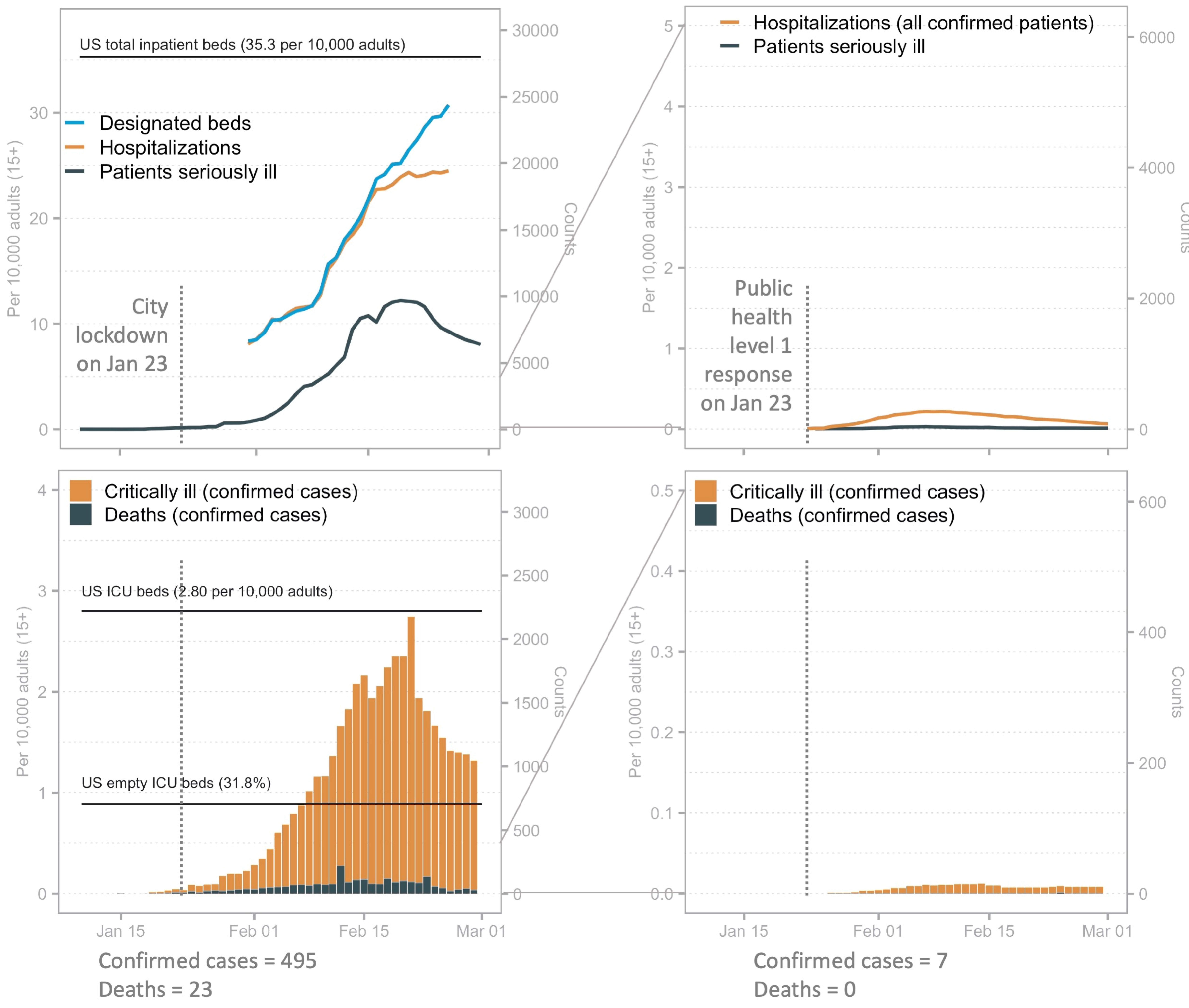

\section{Guangzhou}




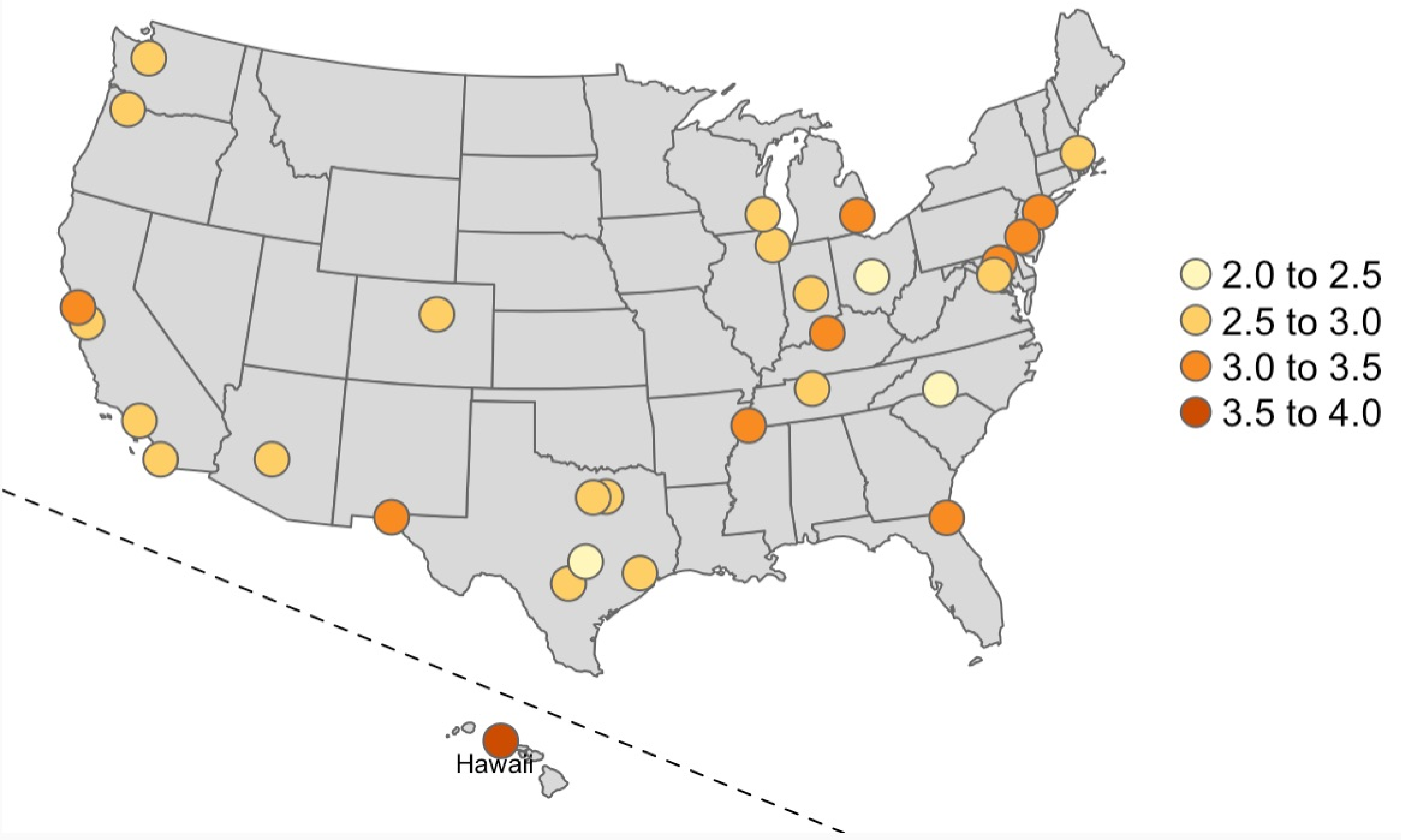




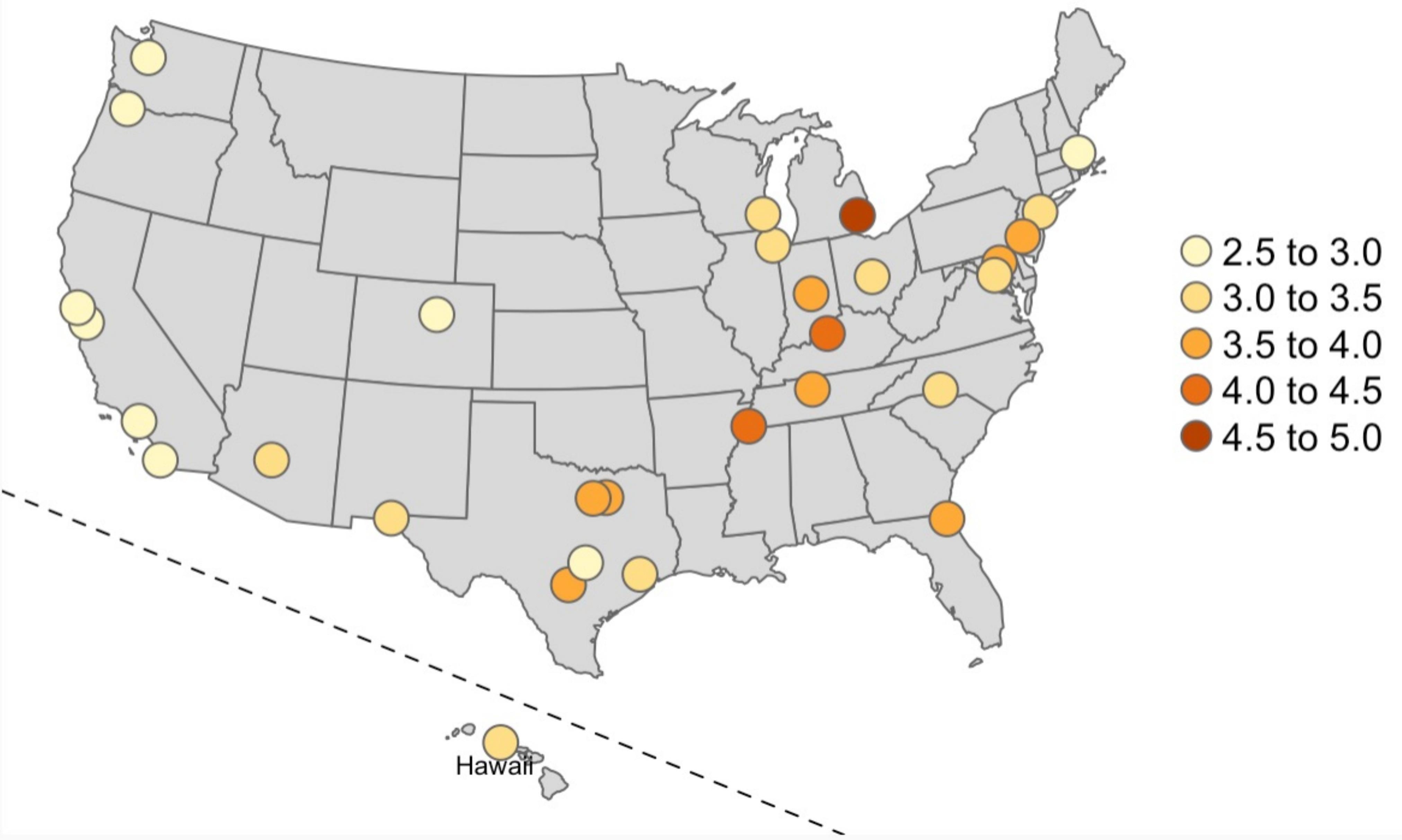

